\title{
Efeito dos ácidos itacônico e fumárico na copolimerização em emulsão do estireno com acrilato de $n$-butila para produção de argamassa cimentícia
}

\section{Effect of itaconic and fumaric acids on the emulsion copolymerization of styrene with n-butyl acrylate to produce cementitious mortar}

\footnotetext{
${ }^{1}$ Laboratório de Síntese de Polímeros, Instituto de Ciência e Tecnologia, Universidade Federal de São Paulo-UNIFESP, São José dos Campos, São Paulo, Brasil. e-mail: sasantoseq@gmail.com, mauricio.pinheiro@unifesp.br

${ }^{2}$ Laboratório de Pesquisa e Desenvolvimento de Polímeros, BASF S.A., Guaratinguetá, São Paulo, Brasil. e-mail: marlon.santos@basf.com
}

\begin{abstract}
RESUMO
Monômeros carboxílicos são frequentemente empregados em formulações de polímeros obtidos via polimerização em emulsão e miniemulsão visando melhorar ás propriedades de aplicação dos látexes. $\mathrm{O}$ objetivo deste trabalho foi avaliar o efeito dos ácidos itacônico IAI), fumárico (AF) e metacrílico (AMA) na copolimerização em emulsão do estireno (STy) com o acrilato de $n$-butila (ABu), visando a obtenção de látexes carboxilados estáveis e com baixa concentração de poli(ácido carboxílico) na fase aquosa para serem empregados como aditivo na preparação de argamassa cimentícia. Látexes de STy/ABu/AI, STy/ABu/AF e STy/ABu/AMA foram obtidos e caracterizados em relação ao teor de sólidos, diâmetro de partícula, polidispersão, viscosidade, temperatura mínima de formação de filme (TMFF), estabilidade ao gelo-degelo, estabilidade coloidal e distribuição dos grupos carboxílicos. Argamassas cimentícias foram preparadas empregando os látexes produzidos. Os resultados obtidos mostram que o tipo de ácido carboxílico empregado afeta as propriedades físico-químicas dos látexes e a interação com os componentes da argamassa. Dentre os ácidos estudados, o AMA apresentou menor efeito nas propriedades coloidais dos látexes, enquanto que o látex produzido com o $\mathrm{AF}$ apresentou baixa estabilidade coloidal, elevada concentração de grupos carboxílicos na fase aquosa e baixa interação com os componentes da argamassa.
\end{abstract}

Palavras-chave: Polimerização em emulsão, látex carboxílico, ácido itacônico, ácido fumárico, argamassa cimentícia.

\begin{abstract}
Carboxylic monomers are often employed in polymer formulations obtained by emulsion or miniemulsion polymerization with the aim of improve the better latex application properties. The aim of this work was to evaluate the effect of itaconic (IA), fumaric (FA) and methacrylic (MAA) acid on the emulsion copolymerization of styrene (STy) with $n$-butyl acrylate $(\mathrm{ABu})$, aiming to obtain stable latexes with low concentration of poly(carboxylic acid) in the aqueous phase, to be used as cement additive. Latexes of $\mathrm{STy} / \mathrm{ABu} / \mathrm{AI}, \mathrm{STy} / \mathrm{ABu} / \mathrm{AF}$ and Sty/ABu/AMA were obtained and characterized in relation to solids content, particle diameter, polydispersity, viscosity, minimum film forming temperature (TMFF), freeze-thaw stability, colloidal stability and distribution of the carboxylic groups. Cementitious mortar was prepared with the latexes and evaluated. The results obtained show that the type of carboxylic acid employed affects the physicochemical properties of latexes and the interaction with the components of the mortar. Among the acids studied, methacrylic acid had a lower effect on the colloidal properties of latexes. The latex produced
\end{abstract}


with fumaric acid presented low colloidal stability, high concentration of carboxylic groups in the aqueous phase and low interaction with the components of the cementitious mortar.

Keywords: Emulsion polymerization, carboxylated latex, cementitious mortar, itaconic acid, fumaric acid.

\section{INTRODUÇÃO}

A técnica de polimerização em emulsão é caracterizada por um sistema onde monômeros insolúveis ou parcialmente solúveis na fase contínua são polimerizados via radicais livres proveniente da decomposição de iniciadores hidrossolúveis. O produto obtido é uma dispersão coloidal de partículas de polímero em água, conhecido como látex [1,2]. No entanto, monômeros solúveis em água, como por exemplo, o ácido acrílico (AA), ácido metacrílico (AMA), acrilamida, ácido itacônico (AI) e ácido fumárico (AF) são tipicamente utilizados na produção de látexes com a finalidade de melhorar a estabilidade coloidal, reduzir a concentração de surfactante e conferir propriedades específicas de aplicação, especialmente, quando empregado como ligante para tintas, adesivos e aditivos para construção civil [3-8].

Considerando as aplicações industriais, um dos fatores indesejáveis na utilização dos monômeros carboxílicos é a polimerização na fase aquosa, com formação de oligômeros. Estes oligômeros são responsáveis por mudanças na viscosidade, variação do $\mathrm{pH}$, baixa resistência à água do filme polimérico e principalmente pela desestabilização do látex durante a polimerização, estocagem e aplicação [3,7,9]. A polimerização dos monômeros carboxílicos na fase aquosa está diretamente relacionada ao coeficiente de partição do ácido carboxílico entre a fase orgânica (gotas de monômero e/ou partículas de polímero) e a fase contínua, que estão relacionadas ao $\mathrm{pH}$ do meio reacional que definirá o grau de ionização destes comonômeros, a composição monomérica e principalmente ao processo de adição do comonômero funcional durante a polimerização $[3,7,9,10]$. A presença de oligômeros de poli(ácido carboxílico) na fase aquosa podem atuar como dispersante, espessante ou plastificante em formulações de argamassa cimentícia, podendo alterar o tempo de cura, a fluidez e a resistência a água da mistura ou do filme polimérico formado $[11,12]$.

Diversos trabalhos foram realizados empregando os ácidos acrílico [3,4,7,9,11-13] e metacrílico [3,7,11,12-17] em polimerizações em emulsão com o objetivo de entender como estes monômeros são incorporados nos polímeros e o efeito destes monômeros nas propriedades finais dos látexes. Ocepek et al. [17] estudaram o efeito de diferentes estratégias de adição dos ácidos acrílico e metacrílico na preparação de látexes carboxilados. De acordo com os autores, as estratégias adotadas resultaram em diferenças significativas no comportamento reológico dos látexes antes e após neutralização. No entanto, poucos trabalhos foram encontrados na literatura sobre o efeito do AI [3,9,10,17-18] e AF [3,21] em polímeros obtidos via polimerização em emulsão. Nos estudos realizados com o AI foi demonstrado que a principal dificuldade está na incorporação do monômero funcional na cadeia polimérica, devido a sua elevada solubilidade em água $[3,10,18,21]$. Em estudos mais recentes foi mostrado que a solubilidade em água destes ácidos, juntamente com o pH do meio reacional e o processo de polimerização têm influência não só na estabilidade coloidal, mas também na cinética da reação, na partição dos comonômeros funcionais e na estabilidade do látex $[3,10]$. No caso do AF, apesar deste ácido ser mais hidrofóbico quando comparado com o AI e AMA, poucos trabalhos foram publicados relatando a utilização do mesmo em reações de polimerização em emulsão e sua aplicação em argamassa cimentícia [3,21].

$\mathrm{O}$ AF e o AI possuem duas carboxilas na molécula, porém o AF é um monômero que possui carboxilas 1,2-di-substituidas dispostas na posição trans, diferenciando do ácido maleico e do AI que possuem corboxilias dispostas na posição cis (Figura 1), além de ser obtido por via química e fermentativa [22]. Considerando a importância industrial e tecnológica destes monômeros carboxílicos e suas aplicações industriais, em especial, na produção de látexes para revestimento de papel e aditivo para argamassa cimentícia, é importante entender e conhecer o efeito destes ácidos carboxílicos, especialmente do AI e do AF na polimerização em emulsão do estireno com acrilato de $n$-butila para aplicação em argamassa cimentícia.

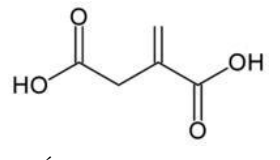

Ácido itacônico

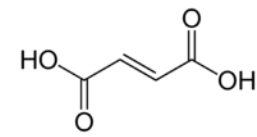

Ácido fumárico

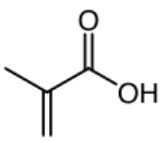

Ácido metacrílico

Figura 1: Estrutura química dos monômeros carboxílicos.

Látexes poliméricos são empregados na preparação de argamassa cimentícia com o objetivo de conferir trabalhabilidade, retenção de água, permeabilidade, estabilidade dimensional, comportamento reológico e resistência mecânica. Alguns trabalhos relatam a influência dos látexes carboxílicos em 
formulações de argamassa cimentícia. Alguns autores descrevem que os látexes carboxilados apresentam efeito positivo na trabalhabilidade da argamassa, reduzindo a demanda por água e auxiliando na retenção de água durante a cura do cimento [11,24]. No entanto, outros autores descrevem que a introdução do látex carboxilado em argamassa cimentícia retarda a hidratação do cimento [12] e aumenta a incorporação de ar devido a presença de emulsificantes e oligômeros na fase aquosa [12,25,26]. De acordo com Wang, et al. [12] o mecanismo de retardação química envolve a complexação dos grupos carboxílicos com íons cálcio presentes no cimento, enquanto que a retardação, está relacionada com a adsorção dos grupos carboxílicos na superfície das partículas. Carbone et al. [25] estudaram o efeito de diferentes látexes poliméricos em pastas de cimento. Foi verificado um efeito negativo na reação de hidratação do cimento com o aumento da relação látex/cimento, porém, com aumento da resistência mecânica e redução da absorção de água. Boutti et al. [26] estudaram a influência da adição de diferentes concentrações de látexes de (STy/ABu) em relação as propriedades de aplicações do cimento. De acordo com os autores, o uso de emulsificante aniônico na etapa de preparação do látex melhora a interação das partículas poliméricas com o cimento devido a presença de forças de repulsão estérica.

Neste sentido, é importante considerar o tipo e a concentração do monômero funcional empregado na síntese e principalmente, a concentração de oligômeros formados na fase aquosa. O objetivo deste trabalho foi estudar a influência do AI e do AF na copolimerização em emulsão do estireno (STy) com acrilato de $n$ butila $(\mathrm{ABu})$, visando obter látexes estáveis e com baixa concentração de grupos carboxílicos na fase aquosa para serem empregados na preparação de argamassa cimentícia. Os resultados obtidos foram comparados com os resultados da polimerização em emulsão do STy com ABu na presença do AMA. O efeito dos monômeros funcionais na conversão global, nas propriedades coloidais, na estabilidade ao gelo-degelo, na distribuição dos grupos carboxílicos e na compatibilidade com argamassa cimentícia foi estudado.

\section{MATERIAIS E MÉTODOS}

\subsection{Materiais}

Os monômeros de estireno (STy), acrilato de $n$-butila ( $\mathrm{ABu}$ ), ácido itacônico (AI) ácido fumárico (AF) e ácido metacrílico (AMA) foram obtidos junto a BASF S.A. Os monômeros de STy, ABu e AMA foram purificados via destilação sob pressão reduzida e armazenados em geladeira a $-4^{\circ} \mathrm{C}$ antes de serem utilizados. O AI e o AF, ambos com grau de pureza igual a 99,9\% foram utilizados sem purificação prévia. Como iniciador de polimerização foi empregado o persulfato de sódio (NaPS, 99,5\%, FMC). Como emulsificante aniônico foi empregado o lauril sulfato de sódio (Disponil ${ }^{\circledR} \mathrm{W} 1 / \mathrm{G}, \mathrm{BASF}$ ) com grau de pureza superior a 99\%. As soluções de $\mathrm{NaOH}$ e $\mathrm{HCl}$ a $0,02 \mathrm{M}$ foram obtidas junto a LabSynth. Como antiespumante, foi utilizado o Dehydran 111 obtido junto a BASF S.A. Para ajuste do pH dos látexes foi empregada a solução de hidróxido de amônia $(10 \% \mathrm{v} / \mathrm{v})$. Em todas as polimerizações foi utilizada água deionizada com condutividade igual a $1,5 \mu \mathrm{S} \mathrm{cm}^{-1}$. Na purificação dos látexes foi empregada a resina de troca iônica Amberlite MB20, obtida junto a Dow Química. Na preparação das argamassas cimentícias, foi empregado o cimento CPII-F da Votoratin e areia de quartzo malhas: 80-100, 120 e 200 mesh.

\subsection{Polimerizações}

As polimerizações foram conduzidas em processo batelada, empregando os monômeros de AI, AF e AMA, com razão mássica igual a 5\% em relação a massa total dos monômeros. Foi empregado um reator de vidro encamisado de 1,0 L, equipado com agitador mecânico, haste âncora, condensador de refluxo e banho termostático para aquecimento e resfriamento do reator. O reator foi carregado com água deionizada $(734,36 \mathrm{~g})$, emulsificante aniônico $(5,0 \mathrm{~g})$ e os monômeros (Tabela 1). A temperatura do meio reacional foi estabilizada em $70^{\circ} \mathrm{C}$ e o iniciador $\operatorname{NaPS}(0,65 \mathrm{~g})$ solubilizado em água deionizada $(60,0 \mathrm{~g})$ foi introduzido no reator. Todas as polimerizações foram conduzidas sob atmosfera de nitrogênio e rotação de $200 \mathrm{rpm}$ por 6 h. Amostras foram retiradas periodicamente do reator em frascos de vidro contendo hidroquinona e colocadas em banho de gelo para posterior análise. Após $6 \mathrm{~h}$ de polimerização, a temperatura do meio reacional foi reduzida para $35^{\circ} \mathrm{C}$ e o látex foi filtrado em malha de $125 \mu \mathrm{m}$. A razão mássica entre os monômeros foi igual a $50 / 50$ e $50 / 45 / 5$. 
Tabela 1: Composições empregadas nas copolimerizações em emulsão do STy/ABu e STy/ABu/ácido carboxílico.

\begin{tabular}{lcccc}
\hline $\begin{array}{c}\text { Monômeros } \\
\text { (g) }\end{array}$ & \multicolumn{4}{c}{ Polimerizações } \\
\cline { 2 - 5 } & $\boldsymbol{S T y / A B u}$ & $\boldsymbol{S T y / A B u / A I}$ & $\boldsymbol{S T y / A B u / A F}$ & $\boldsymbol{S T y / A B u / A M A}$ \\
\hline STy & 100,0 & 100,0 & 100,0 & 100,0 \\
ABu & 100,0 & 90,0 & 90,0 & 90,0 \\
AI & -- & 10,0 & -- & -- \\
AF & -- & -- & 10,0 & -- \\
AMA & -- & -- & -- & 10,0 \\
\hline
\end{tabular}

\subsection{Purificação dos látexes e dos polímeros}

Os látexes foram diluídos com água deionizada em um béquer de 1,0 L para 2,5\% de sólidos. Em seguida, foram adicionados aproximadamente $4 \mathrm{~g}$ da resina de troca iônica e mantido sob agitação mecânica por $1 \mathrm{~h}$. Após $1 \mathrm{~h}$ de agitação, a resina de troca iônica foi removida, a condutividade foi medida e uma nova quantidade de resina $(4 \mathrm{~g})$ foi adicionada e mantida sob agitação por mais $1 \mathrm{~h}$. Este procedimento foi repetido outras 5 vezes até a condutividade constante do látex [3]. Após purificação, os látexes foram precipitados em $100 \mathrm{~mL}$ de metanol sob agitação de $400 \mathrm{rpm}$. Os polímeros precipitados foram lavados com água deionizada e secos em estufa a vácuo na temperatura de $40^{\circ} \mathrm{C}$ por $24 \mathrm{~h}[3,10]$.

\subsection{Preparação e caracterização das argamassas}

As argamassas foram preparadas em uma batedeira planetária de laboratório, sob agitação de $500 \mathrm{rpm}$. A parte líquida (látex, água e antiespumante) foi adicionada na batedeira e após a parte sólida (areia e cimento) foi adicionada lentamente sobre a parte líquida. A parte sólida foi preparada na proporção de 2:1 de areia em relação ao cimento (33,3\% de areia de quartzo malha 100 mesh, $20 \%$ de areia malha 120 mesh, 13,4\% de areia malha 200 mesh e 33,3\% de cimento CPII-F). A relação em massa de polímero/cimento foi fixada em 0,10. A mistura foi mantida sob agitação por 3 min. e vertida no tronco de cone, posicionado no centro da mesa para o teste de abatimento (slamp) [23]. Após 1 min. foi medida a circunferência da massa, tomando-se quatro valores para o cálculo da média.

\subsection{Caracterização dos látexes e dos copolímeros}

$\mathrm{O}$ teor de sólidos foi determinado via analise gravimétrica. Uma alíquota $(\approx 1 \mathrm{~g})$ de cada amostra foi pesada em suportes de alumínio e seca em estufa com circulação de ar a $110^{\circ} \mathrm{C}$ por $1 \mathrm{~h}$. A conversão global foi obtida pela relação entre o teor de sólidos teórico e o teor de sólidos real obtido via análise gravimétrica [9]. $\mathrm{O} \mathrm{pH}$ foi determinado com o auxílio de um $\mathrm{pH}$-metro digital (Micronal, AJX-522). O diâmetro médio de partícula (Dp) e a distribuição de tamanhos de partículas foram determinados por meio da técnica de espalhamento de luz (Malvern, Zetasizer 1000). A viscosidade dos látexes foi determinada por meio de um viscosímetro Brookfield, modelo LVT, com fuso 3 e rotação de $20 \mathrm{rpm}$ a $23^{\circ} \mathrm{C}$.

A estabilidade dos látexes foi avaliada visualmente e por medidas do diâmetro médio de partícula, antes e após $72 \mathrm{~h}$ em estufa a $60^{\circ} \mathrm{C}$. A concentração de coágulos (CC) foi determinada quantitativamente via análise gravimétrica. Os látexes foram pesados $(100 \mathrm{~g})$, diluídos em aproximadamente $500 \mathrm{~mL}$ de água deionizada, filtrados em peneira de aço (malha de $125 \mu \mathrm{m}$ ) e secos em estufa com circulação de ar a $140^{\circ} \mathrm{C}$ por 30 min. Após a secagem, a peneira foi pesada e a concentração de coágulos foi determinada [9]. A estabilidade ao gelo-degelo foi determinada, antes e após neutralização do látex com solução de hidróxido de amônia $(\mathrm{pH}=7,0)$ em geladeira a $-5^{\circ} \mathrm{C}$, por $24 \mathrm{~h}$. As amostras foram avaliadas visualmente em relação à formação de coágulos ou separação de fases. Para as amostras que não apresentaram formação de coágulos ou separação de fases, o ciclo de gelo-degelo foi repetido. Para as amostras que apresentaram separação de fases, o ciclo foi encerrado. As análises de calorimetria exploratória diferencial (DSC) dos copolímeros foram realizadas em um equipamento da marca TA Instruments, modelo DSC 2010. Os copolímeros foram pesados $(10,0 \mathrm{mg})$ em cadinhos de alumínio e as análises conduzidas entre $30^{\circ} \mathrm{C}$ e $200^{\circ} \mathrm{C}$, com taxa de aquecimento de $10^{\circ} \mathrm{C} \mathrm{min}^{-1}$ sob atmosfera de nitrogênio.

A concentração dos grupos carboxílicos na superfície das partículas foi determinada via titulação condutométrica. Após purificação dos látexes via troca iônica, $10 \mathrm{~mL}$ do látex foi transferido para um recipiente encamisado sob agitação magnética e adicionados $5,0 \mathrm{~mL}$ de $\mathrm{NaOH} 0,02 \mathrm{M}$ com vazão de $0,4 \mathrm{~mL}$ $\min ^{-1}$. A mistura foi titulada com solução de $\mathrm{HCl} 0,02 \mathrm{M}$ até um volume total de $8,0 \mathrm{~mL}$, sob atmosfera de $\mathrm{N}_{2}$. Foi empregada uma bureta automática da Metrohm, modelo 665 e um condutivímetro digital (Digimed, modelo DM-31) equipado com eletrodo de platina. A concentração dos grupos carboxílicos no interior e na 
superfície das partículas foi determinada via titulação potenciométrica em meio orgânico. Os copolímeros purificados foram dissolvidos em $100 \mathrm{~mL}$ de dimetilformamida (DMF) e titulados com solução 0,1M de $\mathrm{NaOH}$ em metanol. Provas em branco foram realizadas para determinar a acidez residual do solvente [3,7]. A partir dos resultados obtidos com estas análises foi determinada a concentração dos grupos carboxílicos na superfície das partículas $[\mathrm{COOH}]_{\text {Superfície }}$ Por meio da titulação potenciométrica em meio orgânico foi obtida a concentração dos grupos carboxílicos no interior e na superfície das partículas $[\mathrm{COOH}]_{\text {Superfície + Interior. Por }}$ meio do balanço de massa e de posse da concentração de ácido carboxílido adicionado no reator (Tabela 1), foi determinada a concentração dos grupos carboxílicos na fase aquosa [3,7].

\section{RESULTADOS}

\subsection{Conversão global}

A Figura 2a apresenta as curvas de conversão global em função do tempo para as copolimerizações do $\mathrm{STy} / \mathrm{ABu}$ e STy/ABu/ácidos carboxílicos. As polimerizações conduzidas na presença dos ácidos carboxílicos foram mais rápidas quando comparada com a polimerização conduzida na ausência dos mesmos (STy/BuA). Este efeito de aceleração foi mais significativo para a polimerização na presença do AF, após a primeira hora de polimerização. Para o tempo de polimerização igual a 120 min., a conversão global foi igual a $88 \%$, seguida pelas reações do STy/ABu/AMA e STy/ABu/AI, que apresentaram conversões próximas de 76 e 74 $\%$ respectivamente. Por outro lado, a polimerização conduzida na ausência dos ácidos carboxílicos (STy/BuA) apresentou conversão inferior nas primeiras três horas de polimerização. É importante salientar que a diferença observada na conversão global para a polimerização na presença do AF, foi mais significativa para o intervalo de tempo de 45 a $240 \mathrm{~min}$.
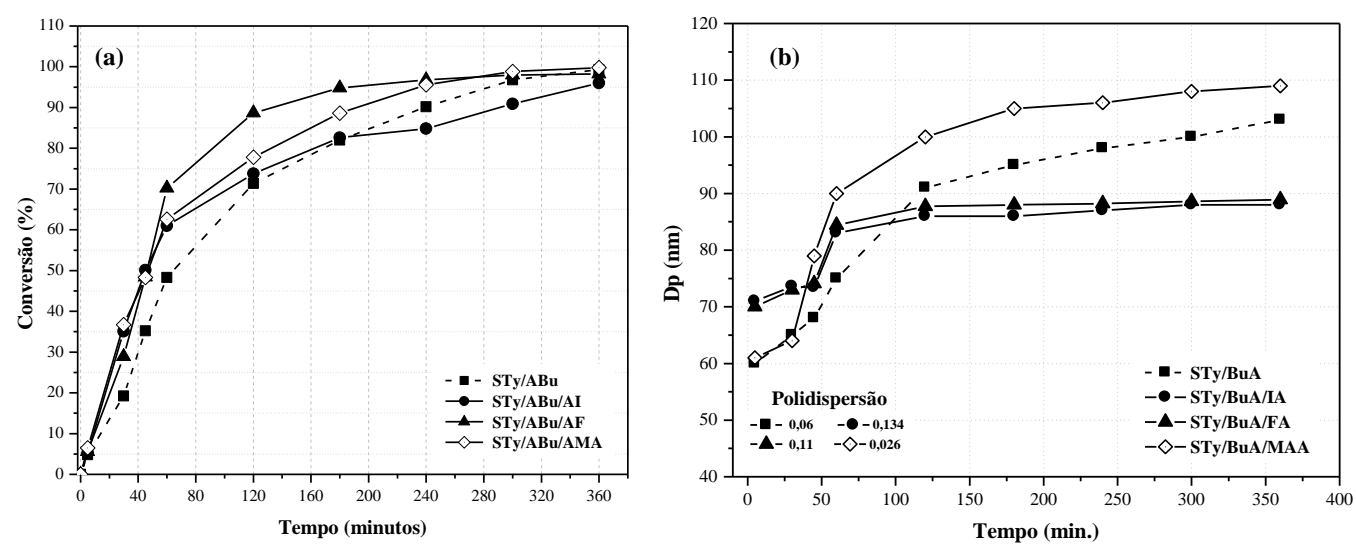

Figura 2: Conversão global (a) e diâmetro médio das partículas (b) em função do tempo para as copolimerizações do $\mathrm{STy} / \mathrm{ABu}$ e STy/ABu/ácidos carboxílicos.

$\mathrm{O}$ aumento na velocidade de polimerização causado pelo AF pode ser justificado pela maior afinidade deste ácido com o STy e com o $\mathrm{ABu}$, menor probabilidade de ocorrer saída de radicais e consequentemente aumento na velocidade de polimerização [5]. Considerando a baixa solubilidade em água deste ácido [3] e sua maior razão de reatividade com o STy $\left(\mathrm{r}_{\mathrm{STy}}=0,056, \mathrm{r}_{\mathrm{FA}=}\right.$ 0,4) [28] este comportamento pode estar relacionado à formação de uma maior concentração de radicais na fase aquosa, com formação de polímero hidrossolúvel. Estes resultados podem ser comprovados analisando as curvas de pH e da condutividade em função do tempo (Figura 3a,b). A natureza iônica do ácido carboxílico na forma dissociada também poderia causar forte influência na taxa de consumo deste comonômero, afetando diretamente a razão de reatividade $\left(\mathrm{r}_{\mathrm{ij}}\right)$. De acordo com a Figura $3 \mathrm{a}$ verifica-se que os valores de $\mathrm{pH}$ estão abaixo do pKa dos ácidos carboxílicos [3]. No caso do AF é possível observar um valor de $\mathrm{pH}$ muito baixo $(\approx 2,15)$ e praticamente constante durante toda a polimerização, o que poderia ser justificado pela homopolimerização do AF na fase aquosa. $\mathrm{O}$ efeito de aceleração da polimerização também pode ser confirmado analisando as curvas do diâmetro médio das partículas (Figura 2b), resultando na formação de um número maior de partículas via nucleação homogênea e/ou heterocoagulação. Um efeito contrário foi observado para a copolimerização do metacrilato de metila com o acrilato de $n$-butila na presença do AF [3]. No presente trabalho, a utilização do AF em um sistema mais hidrofóbico (STy/ABu) resultou no aumento da velocidade de polimerização, nas primeiras horas, podendo ser justificado pela maior afinidade do AF com os comonômeros de base (STy e ABu) [3]. 
A polimerização do STy/ABu/AMA) foi mais rápida quando comparada à polimerização na ausência do AMA (Figura 2a). Este comportamento pode estar relacionado com a afinidade do AMA com os monômeros de base (STy e $\mathrm{ABu}$ ). Como o AMA é menos solúvel em água, uma maior quantidade deste ácido polimerizou no interior das partículas de polímero, resultando na expansão das cadeias poliméricas e ou na presença de seguimentos longos, ricos em grupos carboxílicos na superfície das partículas [3,10,13]. No entanto, é importante ressaltar que o pH do meio reacional ao longo da reação com o AMA, foi próximo ao pKa deste ácido [3], ou seja, nem todas as unidades de COOH estão na forma molecular.
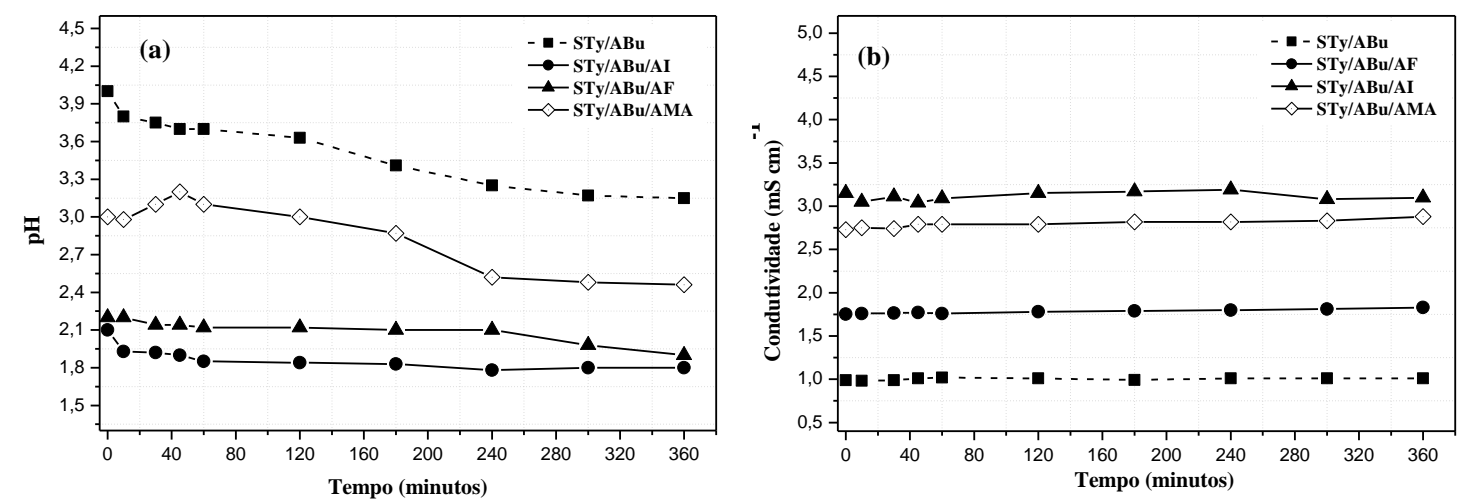

Figura 3: Evolução do pH (a) e da concutividade (b) durante as polimerizações do STy/ABu e STy/ABu/ácidos carboxílicos.

No caso do AI nenhuma diferença na velocidade de polimerização foi observada na primeira hora (Figura 2a). No entanto, após a primeira hora de polimerização verifica-se uma redução da velocidade de polimerização na presença do AF e do AMA. A redução da taxa de polimerização na presença do AI está relacionada com o efeito de impedimento estérico causada pelas carboxilas dispostas na prosição cis na molécula do AI dificultando a propagação das cadeias com este comonômero funcional [29]. Isto significa que um radical do tipo AI dificilmente reage com novas moléculas de AI. Outro aspecto importante está na baixa reatividade do AI com os comonômeros de base $\left(\mathrm{r}_{\mathrm{STy}}=1,36 ; \mathrm{r}_{\mathrm{AI}}=0\right.$ e $\left.\mathrm{r}_{\mathrm{BuA}}=1,10 ; \mathrm{r}_{\mathrm{AI}}=0,23\right)$ [19] e pode ser explicada pela presença de um hidrogênio alílico na molécula do IA, responsável pela formação de radicais muito estáveis $[18,19]$. Neste caso, é pouco provável que um radical do tipo AI inicie a polimerização [18]. Václavová et al. [18] demonstraram que a introdução do AI diminui a taxa de copolimerização do sistema ABu/AI. Resultados semelhantes também foram observados por Ceska [5] na copolimerização em emulsão do estireno/butadieno na presença do AI. Segundo Ceska [5], em quantidades equimolares, a taxa de polimerização obedece a seguinte ordem AMA>AA>. De acordo com os resultados obtidos no presente trabalho e considerando as diferenças na solubilidade em água do AF e do AI, a taxa de polimerização obedece a seguinte ordem $\mathrm{AF}>\mathrm{AMA} \geq \mathrm{AI}$.

As copolimerizações realizadas com o AI e AF apresentaram comportamentos muito semelhantes (Figura 2b). Os valores de Dp para estas reações foram menores em comparação aos valores para a polimerização na ausência dos ácidos carboxílicos. A redução do Dp pode ser justificada pela maior solubilidade em água do AI, resultando na formação de uma maior quantidade de oligoradicais, os quais podem combinar rapidamente para formar novas partículas de polímero ou precipitar na fase aquosa via nucleação homogênea [19]. No caso do AI é descrito na literatura que a presença do AI em reações de copolimerização para sistemas acrílicos-estirenados, aumenta o diâmetro final das partículas e consequentemente, diminui a velocidade de polimerização. No entanto, outros autores afirmam o contrário $[5,18,19]$. Resultados semelhantes aos obtidos no presente trabalho foram descritos para a copolimerização do metacrilato de metila (MMA) com o acrilato de $n$-butila na presença do AI [3] e na copolimerização do $\mathrm{ABu}$ utilizando diferentes concentrações de AI [18]. Por outro lado, nenhum efeito no diâmetro médio das partículas foi observado na copolimerização do STy com diferentes concentrações molares de AI [18].

\subsection{Distribuição dos grupos carboxílicos}

A Figura 4 mostra a distribuição dos ácidos (AI, AF e AMA) no latex (interior das partículas, na superfície das partículas e na fase aquosa). No caso do AMA (látex STy/ABu/AMA) os resultados mostram que $81 \%$ estão presentes no interior das partículas, ficando o restante dividido entre a superfície e a fase aquosa. Estes resultados foram similares aos resultados obtidos por diversos trabalhos publicados, mostrando que o AMA 
polimeriza preferencialmente no interior das partículas de polímero, independente da concentração utilizada [3,7,12-16]. O látex que apresentou menor concentração de grupos carboxílicos na fase aquosa $(9 \% \mathrm{~m} / \mathrm{m})$ foi o látex com o AMA. Apesar do AMA polimerizar preferencialmente no interior das partículas e ser o mais indicado para aplicações industriais, ele é pouco utilizado devido ao custo e disponibilidade quando comparado ao AI e o AF. Neste caso, o estudo e a utilização do AI e do AF em copolimerizações com o STy e o $\mathrm{ABu}$ se tornam muito atraentes.

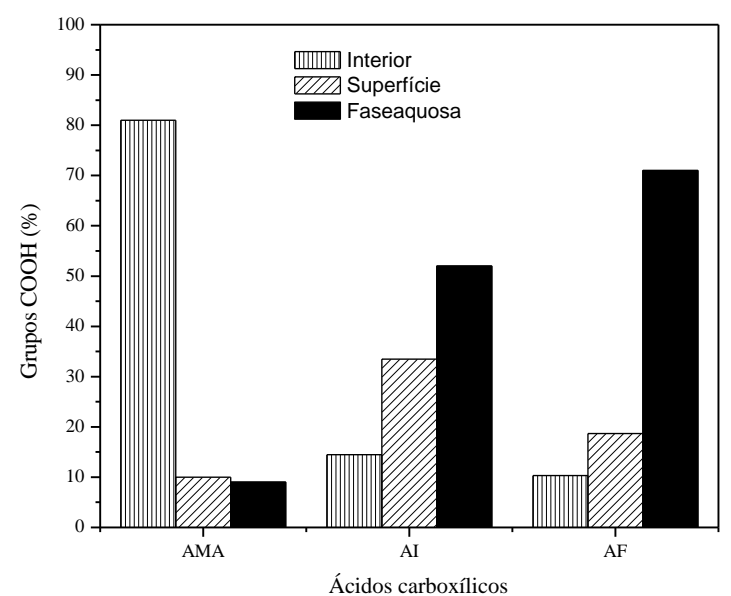

Figura 4: Distribuição dos grupos carboxílicos $(\% \mathrm{~m} / \mathrm{m}$ de grupos $\mathrm{COOH})$ nas diferentes fases do sistema (interior/superfície/fase aquosa) para os látexes funcionalizados com AMA, AI e AF.

A Figura 4 mostra que o látex produzido com o AI possui 33,5\% deste ácido na superfície das partículas, $52 \%$ na fase aquosa e apenas $14,5 \%$ no interior das partículas. Este mesmo comportamento foi observado para a copolimerização do MMA com $\mathrm{ABu}$ na presença do AI [3] e pode ser justificado pelo fato do AI polimerizar preferencialmente na fase aquosa na forma de hidrosolúveis, ou ainda ficar adsorvido na superficie das particulas. É importante ressaltar que os hidrosolúveis adsorvidos são removidos durante a etapa de purificação dos látices pela técnica de troca iônica. Além disso, considerando-se a alta solubilidade em água e a baixa reatividade do AI, parte deste comonômero pode ser encontrado livre na fase aquosa como monômero residual. Estes resultados podem ser confirmados analisando-se o perfil das curvas de variação do pH apresentadas na Figura 3a e da condutividade do látex (Figura 3b). É possível observar também que durante toda a polimerização o $\mathrm{pH}$ do látex preparado com AI foi menor em comparação com as polimerizações realizadas na presença do AMA e do AF. Ceska [5] observou um aumento da concentração de AI na superfície das partículas de polímero para látexes de STy/butadieno/AI com o aumento da concentração de AI. Vijayendran [10] e Fordyce et al. [21] observaram que a concentração de grupos carboxílicos no interior das partículas de polímero aumentou significativamente com o incremento da concentração do AI, quando copolimerizado com o STy.

No caso do látex obtido com o $\mathrm{AF}$ (STy/ABu/AF) os resultados mostram uma baixa concentração de grupos carboxílicos no interior $(10,3 \%)$ e na superfície das partículas $(18,7 \%)$ e uma grande quantidade na fase aquosa $(71,0 \%)$. Este resultado pode ser uma indicação da polimerização do AF na fase aquosa, com formação de hidrossolúveis ou mesmo, na forma de monômero residual, o que pode ser explicado pelo menor valor do $\mathrm{pH}$ e da condutividade no decorrer da polimerização (Figura 3a,b). Considerando-se a baixa solubilidade em água do AF [3], sua elevada concentração na fase aquosa só poderia ser justificada por uma diferença muito significativa nas razões de reatividade entre este ácido e as moléculas de STy e ABu. Comportamento semelhante foi observado para o sistema MMA/ABu/AF, onde 7,7\% do AF foi encontrado na superficie das partículas [3]. Em um estudo de copolimerização de STy com os ácidos AA e AF foi constatado que apenas $18,6 \%$ estava na superfície das partículas [8]. Os resultados destes dois estudos foram justificados pela diferença entre as características hidrofílicas e hidrofóbicas do AF na forma ionizada e pela repulsão eletrostática entre os grupos carboxílicos.

\subsection{Estabilidade gelo-degelo e Tg}

A Tabela 2 mostra os resultados obtidos nos testes de estabilidade ao gelo-degelo para os látexes antes e após o ajuste do $\mathrm{pH}$. Os resultados indicam maior estabilidade dos látexes preparados com AMA e AI, apresentando estabilidade ao gelo-degelo de 3 ciclos antes da neutralização e de 5 e 4 ciclos após neutralização $(\mathrm{pH}=7,0)$. Por outro lado, o látex obtido sem os monômeros carboxílicos (STy/ABu) apresentou estabilidade ao gelo-degelo de 1 ciclo, não sendo verificada nenhuma melhora na estabilidade com o aumento 
do pH. Já o látex STy/ABu/AF apresentou o pior resultado de estabilidade, coagulando após o primeiro ciclo de estabilidade ao gelo-degelo. Este mesmo comportamento foi observado para o látex neutralizado $(\mathrm{pH}=7,0)$. No caso do látex obtido com o $\mathrm{AF}$, o pH final foi igual a 1,9 e inferior ao pKa do AF. Este valor de pH é uma condição que desfavorece a estabilização das partículas para baixas concentrações de ácido carboxílico na superfície das partículas. Resultados semelhantes foram observados para os copolímeros de poli(MMA/ABu/ácidos carboxílicos [3].

Comparando os resultados da estabilidade ao gelo-degelo do sistema (STy/ABu/ácidos carboxílicos) antes e após neutralização é possível observar um aumento da estabilidade ao gelo-degelo para os látexes com AI (STy/ABu/AI) e com o AMA (STy/ABu/AMA). Este comportamento está relacionado à concentração de grupos carboxílicos na superfície das partículas de polímero. A concentração de $\mathrm{COOH}$ na superfície das partículas para o látex $\mathrm{STy} / \mathrm{ABu} / \mathrm{AF}$ foi maior quando comparada com a concentração de $\mathrm{COOH}$ para o látex funcionalizado com AMA (Figura 4). É possível correlacionar os resultados de distribuição dos grupos carboxílicos com a estabilidade ao gelo-degelo e principalmente com o $\mathrm{pH}$ dos látexes. O látex obtido na presença do AMA foi o que apresentou maior concentração de grupos carboxílicos no interior das partículas e consequentemente, maior estabilidade ao gelo-degelo quando comparado aos latexes produzidos com AI e AF. Um comportamento semelhante foi observado por Guziak et al. [30] para látexes de STy/AMA, obtidos com diferentes concentrações de AMA. Os autores observaram um aumento da estabilidade ao gelo-degelo com o aumento da concentração de grupos carboxílicos no interior das partículas de polímero.

A Tabela 2 também mostra os resultados obtidos da $\mathrm{Tg}$ dos copolímeros com diferentes ácidos carboxílicos. Verifica-se um pequeno aumento da $\mathrm{Tg}$ para os copolímeros obtidos na presença dos ácidos carboxílicos, quando comparada com a Tg do copolímero na ausência dos ácidos carboxílicos. O aumento mais significativo da $\mathrm{Tg}$ foi observado para o copolímero produzido com o AMA ( $\left.\mathrm{Tg} \approx 26,5^{\circ} \mathrm{C}\right)$. Já os copolímeros obtidos com os ácidos AI e AF a variação foi pouco significativa, apresentando valores da $\mathrm{Tg}$ de $\approx 24,8{ }^{\circ} \mathrm{C}$ para o STy/ABu/AF e $25,0{ }^{\circ} \mathrm{C}$ para o STy/ABu/AI.

Tabela 2: Temperatura de transição vítrea ( $\mathrm{Tg})$ dos copolímeros e estabilidade ao congelamento e descongelamento dos látexes.

\begin{tabular}{lccc}
\hline \multirow{2}{*}{ Copolímeros } & \multirow{2}{*}{$\mathbf{T g}\left({ }^{\mathbf{0}} \mathbf{C}\right)$} & \multicolumn{2}{c}{ Estabilidade $($ ciclos $)$} \\
\cline { 3 - 4 } & 22,0 & $\boldsymbol{p H}<\boldsymbol{p} \boldsymbol{K a}$ & $\boldsymbol{p H ~ 7 , 0}$ \\
\hline STy/ABu & 25,0 & 3,0 & 1,0 \\
STy/ABu/AI & 3,0 & 4,0 \\
STy/ABu/AF & 24,8 & 1,0 & 1,0 \\
STy/ABu/AMA & 26,0 & 3,0 & 5,0 \\
\hline
\end{tabular}

As análises de Tg foram realizadas com os copolímeros purificados [3] e apesar da pequena diferença observada entre os valores é possível sugerir que o menor valor da Tg para o copolímero com AF está relacionada com a baixa concentração do AF incorporado na cadeia do polímero (Figura 4). No caso do copolímero obtido na presença do AMA houve uma maior incorporação do AMA na cadeia do copolímero e, consequentemente, um maior valor da $\mathrm{Tg}$. Este aumento de $\approx 4^{\circ} \mathrm{C}$ na $\mathrm{Tg}$ está relacionado com a incorporação deste ácido na cadeia do polímero e devido a Tg do poly(AMA) ser próxima de $162^{\circ} \mathrm{C}$ [1,17]. Resultados semelhantes foram obtidos por Santos et al. [7] para os copolímeros de STy/ABu na presença do AMA e Koh et al. [16] para copolímeros de ABu com AMA. De acodo com os autores, a Tg dos copolímeros é influênciada pela concentração de grupos carboxílicos no interior das partículas de polímero.

\subsection{Interação com os componentes da argamassa}

A Figura 5 mostra o aspecto e o comportamento das argamassas preparadas com os látexes após o teste de abatimento (slamp). Após a remoção do tronco de cone, foi realizado o teste de abatimento e as medidas das circunferências foram realizadas após $1 \mathrm{~min}$. $\mathrm{O}$ menor diâmetro foi obtido para a argamassa preparada com o látex de STy/ABu. Foi observado também, boa compatibilidade com os componentes da argamassa, sem a presença de flotação de água e separação de fases (Figura 5a). O maior diâmetro foi obtido para a argamassa produzida com o látex na presença do $\mathrm{AI}$ (STy/ABu/AI), apresentando boa trabalhabilidade, pouca flotação de água e pequena separação de fases (Figura 5c). O mesmo comportamento foi observado para a argamassa preparada com o látex produzido com o AMA (Figura 5b). Já a argamassa preparada com o látex produzido com o AF, a mesma não apresentou fluidez e trabalhabilidade, não sendo possível efetuar o teste de "slamp" pois a argamassa estava seca (Figura 5d). Estes resultados mostram o efeito do tipo de ácido e da distribuição 
dos grupos carboxílicos nas diferentes fases do látex (interior, superfície das partículas e fase aquosa) e sua influência no comportamento da argamassa cimentícia. A presença de grupos carboxílicos na fase aquosa, na forma de poli(ácido carboxílico) pode resultar na complexação dos íons $\mathrm{Ca}^{+2}$ do cimento, resultando em desestabilização da argamassa e retardação da cura do cimento [12,26]. As espécies de poli(AF) presentes na em grande quantidade na fase aquosa podem atuar como dispersante, porém, a trabalhabilidade da argamassa é uma propriedade que depende da concentração e da interação destas espécies com os compenetes da argamassa. Látexes com característica aniônica apresentam maior efeito de retardação da cura do cimento, porém, para partículas poliméricas com alta densidade de carga este efeito aumenta [12].

Comparando os diâmetros obtidos após teste de slump, a diferença de 22,0 cm (látex STy/ABu) para $25,0 \mathrm{~cm}$ (látex Sty/ABu/AI) está relacionada à presença dos grupos carboxílicos e principalmente a densidade de carga na superfície das partículas. Quando o látex é misturado com os componentes da argamassa, as partículas de polímero carregadas negativamente são dispersas de forma homogênea no meio e aderem diferentemente na superfície dos agregados, auxiliando ou dificultando a dispersão [26,31].

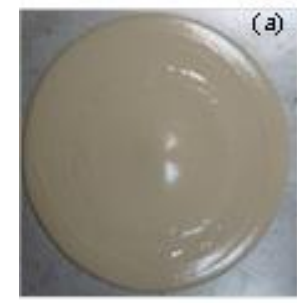

$\mathrm{d}=22,0 \mathrm{~cm}$

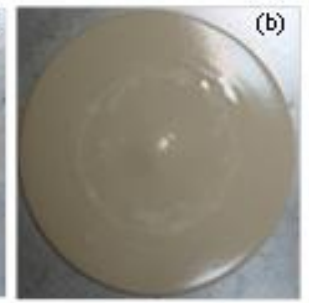

$\mathrm{d}=24,0 \mathrm{~cm}$

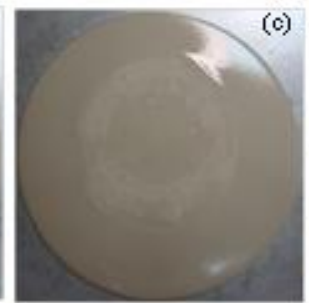

$\mathrm{d}=25,0 \mathrm{~cm}$

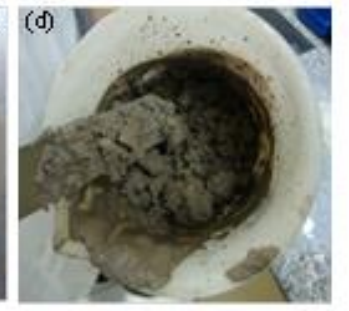

Nẫo foi possivel medir

Figura 5: Diâmetro e aparência das argamassas preparadas com os látexes após o teste de abatimento (slamp). (a) látex $\mathrm{STy} / \mathrm{ABu}$, (b) látex STy/ABu/AMA, (c) STy/ABu/AI e (d) STy/ABu/AF.

\section{CONCLUSÕES}

De acordo com os resultados obtidos, a introdução dos ácidos carboxílicos nas polimerizações em emulsão do STy com ABu afeta as propriedades físico-químicas dos látexes e das argamassas cimentícias. A presença dos comonômeros funcionais de AI, AF e AMA afetou a conversão global e a fluidez da argamassa. Na presença do $\mathrm{AF}$ a polimerização foi mais rápida, enquanto que a polimerização conduzida na presença do $\mathrm{AI}$ foi mais lenta. Os ácidos carboxílicos utilizados neste trabalho tiveram influência no crescimento das partículas de polímero quando comparada com a copolimerização conduzida na ausência destes ácidos. No caso do AMA verificou-se um aumento do diâmetro médio das partículas, enquanto que na presença do AI e do AF foi observada uma redução do diâmetro médio das partículas.

Os ácidos carboxílicos utilizados neste estudo ficaram diferentemente distribuídos entre a fase aquosa, interior das partículas e superfície das partículas. No caso do AI a distribuição foi mais homogênea quando comparada com a distribuição dos outros ácidos. Contrário ao esperado, o AF foi encontrado em grande quantidade na fase aquosa, enquanto o AMA foi encontrado em grande quantidade no interior das partículas.

Os látexes obtidos com os ácidos AMA e AI apresentaram maior estabilidade ao congelamento e descongelamento, onde o látex funcionalizado com o AMA foi mais estável. Nenhuma melhora na estabilidade ao gelo-degelo foi observada para o látex funcionalizado com AF, mesmo após a neutralização $(\mathrm{pH}=7,0)$. Foi observada uma variação de $4,0-5,0^{\circ} \mathrm{C}$ na $\mathrm{Tg}$ dos copolímeros.

$\mathrm{O}$ menor diâmetro da circunferência da argamassa foi obtido na presença do látex de STy/ABu, apresentando boa compatibilidade com os componentes da argamassa. O maior diâmetro foi obtido para a argamassa produzida com o látex de STy/ABu/AI, apresentando boa trabalhabilidade, pouca flotação de água e pequena separação de fases. A argamassa preparada com o látex produzido com o AF não apresentou boa fluidez e trabalhabilidade, não sendo possível efetuar o teste de "slamp". A presença dos grupos carboxílicos na fase aquosa, na forma de poli(ácido carboxílico) afetou a trabalhabilidade da argamassa cimentícia.

\section{AGRADECIMENTOS}

Os autores agradecem a FAPESP (processo 2013/25619-3) e a BASF S.A. pela bolsa, doação dos reagentes e caracterizações.

\section{BIBLIOGRAFIA}


[1] LOVELL, P.A., EL-AASSER, M.S. Emulsion Polymerisation and Emulsion Polymers, Wiley, Chichester, 1997.

[2] WARSON, H., FINCH, C.A. Applications of Synthetic Resin Latices, v. 3, Wiley, Chichester, 2001.

[3] OLIVEIRA, M.P., GIORDANI, D.S., SANTOS, A.M. "The role of itaconic and fumaric acid in the emulsion copolymerization of methyl methacrylate and n-butyl acrylate", European Polymer Journal, v. 42, pp. 1196-1205, 2006.

[4] VORWERG, L., GILBERT, R.G.,"Electrosteric stabilization with poly(acrylic) acid in emulsion polymerization: Effect on kinetics and secondary particle formation", Macromolecules, v. 33, pp. 66936703, 2000.

[5] CESKA, W.G., "Carboxyl-stabilized emulsion polymers", Journal of Applied Polymer Science, v. 18, pp. 2493-2499, 1974.

[6] SAKOTA, K., OKAYA, T. "Polymerization behavior and distribution of carboxyl groups in preparation of soap-free carboxylated polystyrene latexes", Journal of Applied Polymer Science, v. 21, p. 1035-1043, 1977.

[7] SANTOS A.M., MCKENNA T.F., GUILLOT J. "Emulsion copolymerization of styrene and $n$-butyl acrylate in presence of acrylic and methacrylic acids: Effect of $\mathrm{pH}$ on kinetics and carboxyl group distribution", Journal of Applied Polymer Science, v. 65, pp. 2343-2355, 1997.

[8] SAKOTA, K., OKAYA, T. "Polymerization behavior of carboxylic monomers in the preparation of carboxylated latexes", Journal of Applied Polymer Science, v. 20, pp. 2583-2587, 1976.

[9] OLIVEIRA, M.P., SILVA, C.R. GUERRINI, L.M., "Effect of itaconic acid on the wet scrub resistance of highly pigmented paints for architectural coatings", Journal of Coatings Technology Research, v.8, pp.439447, 2011.

[10] VIJAYENDRAN, B.R. "Effect of carboxylic monomers on acid distribution in carboxylated polystyrene lattices", Journal of Applied Polymer Science, v.23, pp. 839-901, 1979.

[11] WANG, R., WANG, P.M. "Function of styrene-acrylic ester copolymer latex in cement mortar", Materials and Structures, v.43, pp. 443-451, 2010.

[12] KONG, X., PAKUSCH, J., JANSEN, D., EMMERLING, S., et al., "Effect of polymer latexes with cleaned serum on the phase development of hydrating cement pastes", Cement and Concrete Research, v.84, pp. 30-40, 2016.

[13] SHOAF, G., PHOEHLEIN, W.G., "Kinetics of emulsion copolymerization with acrylic acids", J. Appl. Polym. Sci., v. 42, pp. 1213-1237, 1991.

https://doi.org/10.1002/app.1991.070420506 [14] SLAWINSKI, M., SCHELLEKENS, M.A.J., MEULDIJK, J., et al., "Seeded emulsion polymerization of styrene: Influence of acrylic acid on the particle growth process", Journal of Applied Polymer Science, v. 76, pp. 1186-1196, 2000.

[15] GUILLAUME J.L., PICHOT C, GUILLOT J, "Emulsifier-free emulsion copolymerization of styrene and butyl acrylate. II. Kinetic studies in the presence of ionogenic comonomers", Journal of Polymmer Sci., Part A: Polymer Chemistry, v. 26, pp. 1937-1959, 1988.

[16] KOH, A.Y.C., MANG, S., BOTHE, M., et al., "The influence of copolymerization with methacrylic acid on poly(butyl acrylate) film properties", Polymer, v.47, pp. 1159-1165, 2006.

[17] OCEPEK, M., BERCE, P., RAZBORSEK, T., et al., "Influences of feeding strategies on AA and MAA carboxylated latexes" Journal of Applied Polymer Science, DOI: 10.1002/app.42062, 2015.

[18] VACLAVOVA, E., HRIVIK, A., CHRASTOVA, V. "Emulsion copolymerization of styrene and butyl acrylate with itaconic acid", Makromolecukes Chemistry, v.193, pp.2243-2250, 1992.

[19] LOCK, M.R., EL-AASSER, M.S., KLEIN, A., et al., "Role of Itaconic acid in latex particle nucleation”, Journal of Applied Polymer Science, v. 42, pp.1065-1072, 1991.

[20] ABDOLLAHI, M., "Effect of carboxylic acid monomer type on particle nucleation and growth in emulsifier-free emulsion copolymerization of styrene-carboxylic acid monomer", Polymer Journal, v.39, pp.802, 2007.

[21] FORDYCE, R.G., HAM, G.G. "Copolymerization II. The mechanism of emulsion copolymerization of styrene and itaconic acid”, J. Am. Chem.Sci., v.69, pp. 695-696, 1947. 
[22] KLEMENT, T., BÜCHS, J. "Itaconic acid. A biotechnological process in change", Bioresource Technology, v.135, pp. 422-431, 2013.

[23] ASSOCIAÇÃO BRASILEIRA DE NORMAS TÉCNICAS (ABNT), NBR NM 67: Concreto Determinação da consistência pelo abatimento do tronco de cone. Rio de Janeiro, 8p, 1998.

[24] PEI, M., KIM, W., HYUNG, W., et al., "Effects of emulsifiers on properties of poly(styrene-butyl acrylate) latex-modified mortars", Cement and Concrete Research, v.32, pp. 837-841, 2002.

[25] CARBONE, C. E., SANTOS, H., ROMANO, R.C.O., et al., "Efeito da adição de látex nas pastas de cimento branco no estado endurecido", Ambiente Construído, v.13, pp. 317-330, 2013.

[26] BOUTTI, S., URVOY, M., DUBOIS-BRUGGER, I., et al., "Influence of low fractions of styrene/butyl acrylate polymer latexes on some properties of ordinary portland cement mortars" Macromolecular Materials Engineering, v. 292, pp.33-45, 2007.

[27] BAJAJ, P., MEENAKSHI, G., CHAVAN, R.B. "Synthesis and characterization of methacrylic acidethyl acrylate copolymers" Journal of Applied Polymer Science, v.73, pp.1771-1783, 1994.

[28] SWITALA-ZELIAZKOV, M. "Radical copolimerization of fumaric acid with styrene in DMF solution" European Polymer Journal, v.35, pp.1591-1597, 1999.

[29] [29] ASKAROV, M.A., SEMENOVA, I.L.N., "Fiz. Khimo Prirodn. I Sintetich Polimerov”, Inst. Khim. Polimerov, v.1, p.177,1962.

[30] GUZIAK, F.F., MACLAY, W.N. "Redispersible latex polymer" Journal of Applied Polymer Science, v.7, pp. 2249, 1963.

[31] CHANDRA, S., OHAMA, Y. Polymers in Concrete, CRC Press, Boca Raton, pp. 83-113,1994.

ORCID

Marlon Sandro dos Santos

https://orcid.org/0000-0002-7810-7647

Maurício Pinheiro de Oliveira https://orcid.org/0000-0002-8210-5134

Samuel Augusto dos Santos https://orcid.org/0000-0003-0100-1219 